\section{Experimental physics, experimental art}

\section{What happens when artists and particle physicists are brought together to exchange ideas? Ken McMullen describes the creative fallout.}

T he concept of Signatures of the Invisible was sparked by a chance discussion I had with Maurice Jacob, high up in the Alps, on a snowy track in Briançon, France. It was December 1996, and I had got to know Maurice, then head of the Theory Division at CERN, Europe's particle-physics laboratory, through a social network entirely independent of science.

Rather than a movement, Signatures of the Invisible became a discourse between artists and particle physicists. It led to the creation of works of art that have been exhibited internationally from Beijing to New York, and have been seen by over a million people. In line with Signature's general philosophy, the works are not illustrative of science as such, but are responses - conveyed in various media and conceptual frameworks - to this discourse between artists and scientists. If Signatures of the Invisible had any manifesto at all, it was that it should not conform to any single artistic dogma.

Maurice is a man of wide cultural interests, and his generosity in taking time to describe to an outsider, an artist, what went on at CERN opened a door in my consciousness. At 1,500 metres above sea level, we conceived the idea of bringing together artists and particle physicists, and seeing what creativity would fall out.

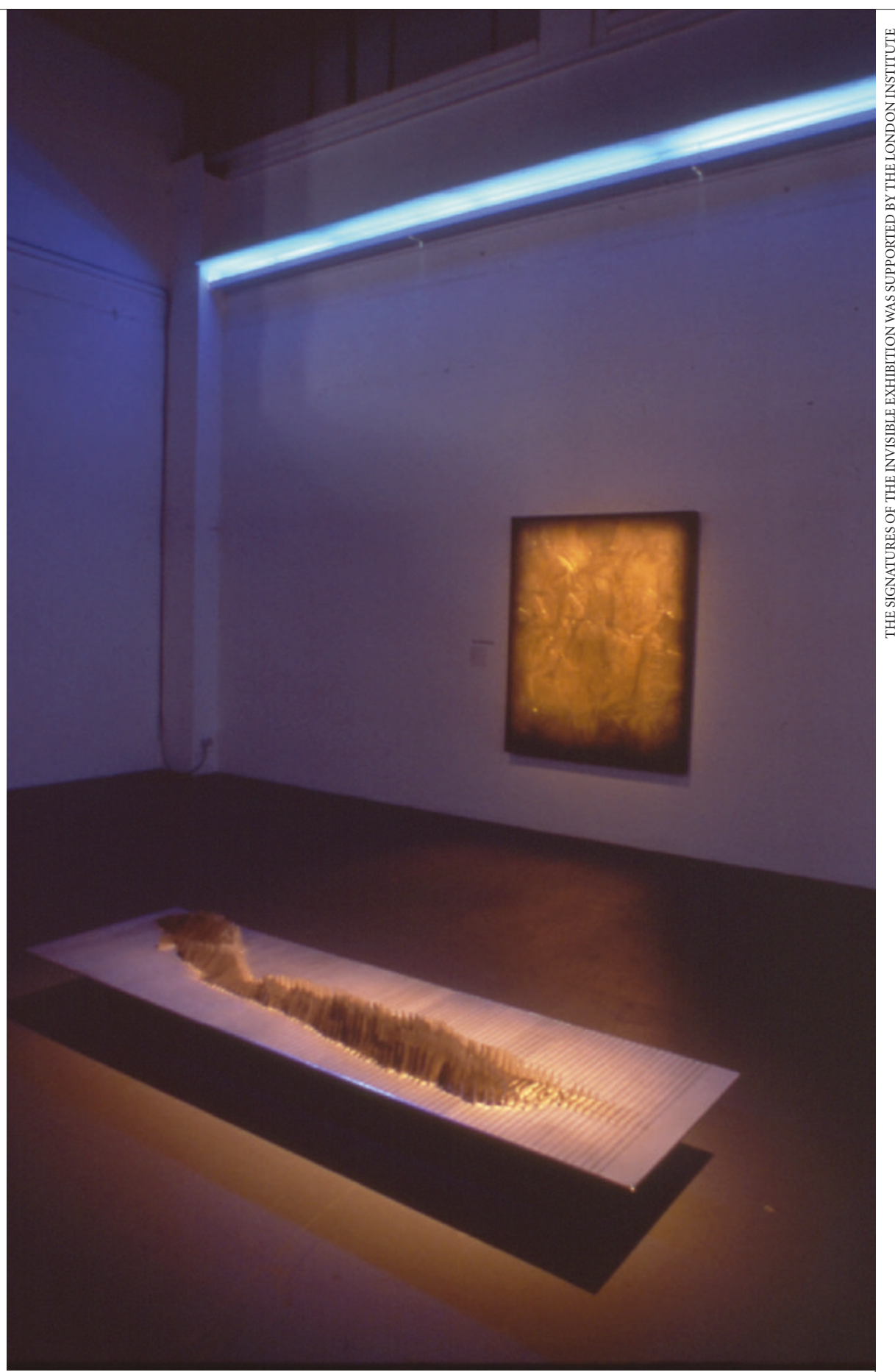

Creativity ruffled: McMullen's work Skin Without Skin emerged from a discussion of crumple theory.

Within months of meeting, Maurice and I had arranged for this to happen at CERN in Geneva. A group of 12 leading European artists were offered free access to CERN's remarkable thinkers and experimenters for discussions about the strange new world of high-energy physics. It took "Artistic practice cannot another two years to organize derive from the artist's funding, but following this, the subjectivity alone, but 12 of us went to spend a week demands engagement or so - some of us much between the artist's longer - at the CERN laboraintra-psychic processes tory. High levels of technical and the external world." assistance helped us to produce works addressing what, as we came to recognize through our discussions, are some of the most profound questions confronting people today.

Signatures of the Invisible was motivated by the belief that artistic practice, if it is to have meaning in the modern world, cannot derive from the artist's subjectivity alone, but demands engagement between the artist's intra-psychic (subjective or intuitive) processes and the external world in which we live. Until this point, little had been done by artists to address the insights offered by particle physics - quantum mechanics, relativity, matter and antimatter - into the underlying laws of nature. 
Particle physics is of course one of the most demanding intellectual disciplines, and artists by their nature and training do not work in the same way. But artists have their own disciplines and emphases, their own ways of looking at things, which are equally valid and can offer corresponding insights into the nature of things. Take colour: for the artist it is a purely visual phenomenon, but for physicists it can relate to temperature, extending from the infrared to the ultraviolet and beyond. Take surface: for an artist, a surface is where you place things - paint on a canvas surface for example - while for the physicist, it is the outermost part of a body, and may be associated with levels of energy. Take units of time: for the film-maker it is 24 frames per second; for a physicist it may be a matter of femtoseconds.

Signatures of the Invisible was not, then, a meeting of opposites, but a correspondence between equal partners from different traditions. It was not a straightforward illustrative 'sci-art' project, but a free flow of ideas between groups of people, each concerned in their own way with the mystery and the reality of what makes us who we are; each trying to understand the Universe in which we find ourselves a part.

During the three years of engagement with CERN, I found myself in an unknown world, which while offering a unique opportunity to explore new territory, also challenged many previously held certainties. The journey was exciting and at times terrifying as far as my own artistic practice was concerned. There were many periods when I was completely lost. There were also moments of exhilaration when some works found their form and occasionally their resolution.

At one point I found myself in freewheeling discussions with John March Russell, a CERN theorist, about the physics involved in crumpling a piece of paper: I discovered there is actually a 'crumple theory'. We began to talk of the surface as a concept, the kind of energy that is released in the act of crumpling, and the strange fact that a crumple can never be repeated exactly even if the paper is the same construction right down to the atomic level, and the same energy and force are applied. So the crumples are probably a concrete signature of time. Fascinated, I continued these discussions at highly technical levels with CERN

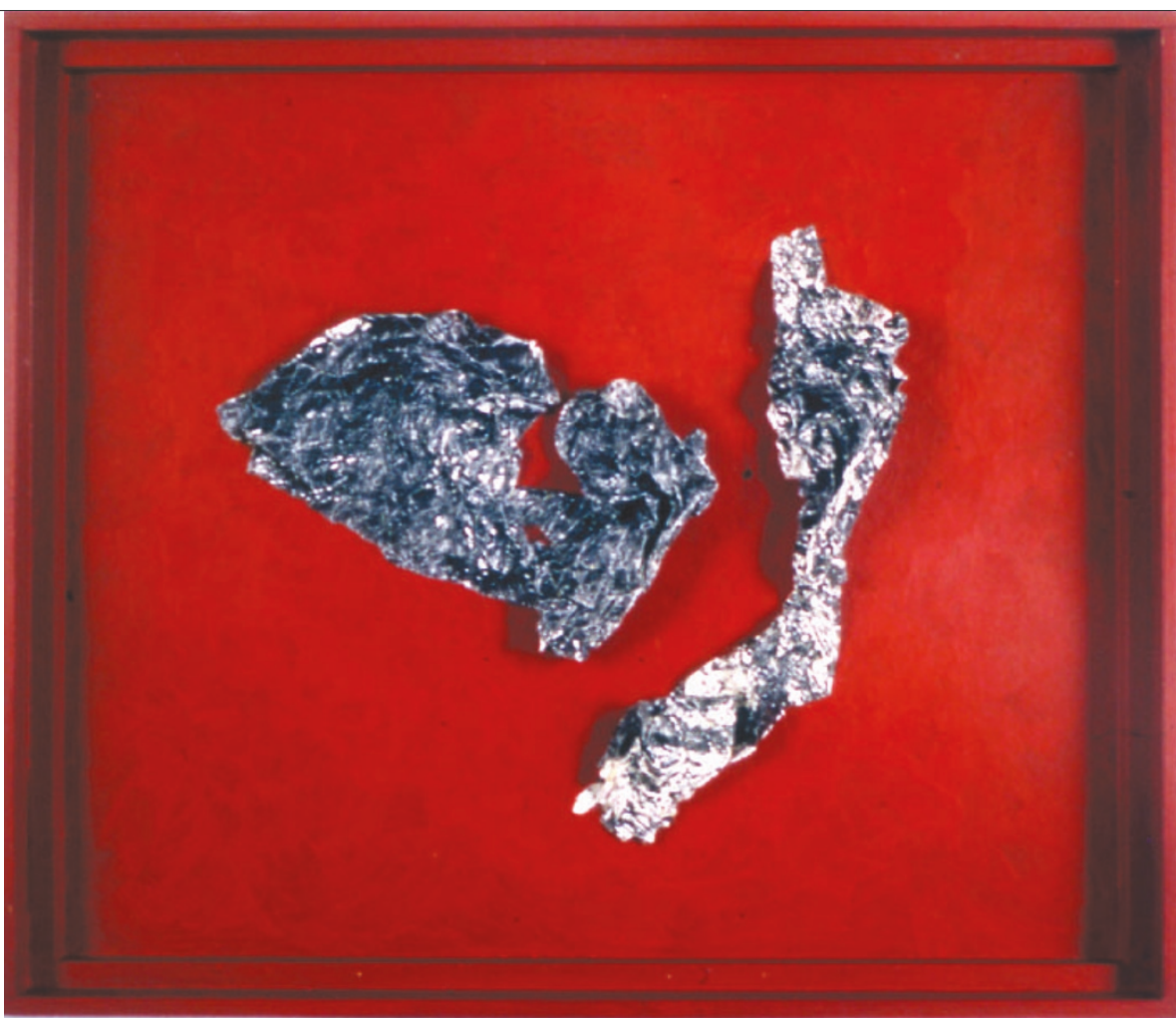

A mark of time: the crumple on the right became the sketch for Skin Without Skin.

engineers who helped me to experiment for months with the theory: crumpling many different pieces of paper, and eventually crumpling giant sheets of titanium. We used laser scanning, laser cutting, laser welding - high-tech tools that I would otherwise never have come to use in my work.

The Signatures philosophy carried the risk of complete failure, of course, and some ideas did fail. But the exhibition that Signatures spawned, of over 50 diverse artworks, showed the overall success of this encounter. Several of us are now engaged in vigorous exploration of dark matter and dark energy, and the consequences of these discoveries, at the Stanford Linear Accelerator Center (SLAC) in California.

Ken McMullen is an artist and film-maker, and research professor at London's University of the Arts.

\section{TAPE TRANSCRIPT FROM A CERN ENCOUNTER}

John March Russell If I think about the interaction of two particles, I think of two lines meeting... There's this strange thing called 'a spacefilling curve'... You think of space being bigger than a line or a surface being bigger than a line but actually ... in a sense they have the same number of points.

Ken McMullen I like that... I like that a lot.

JMR If I draw a plane and count the number of points on that plane, there are actually the same number of points as if I'd drawn a line... This seems counter intuitive but both are actually infinite. A space-filling curve does crazy things... it's so bendy that it fills up the surface or volume completely.
KM The line would have to cross itself though.

JMR No, you can do this mathematically... It twists round in a very complicated way... There are people here who could demonstrate this.

KM If we do these surfaces with lines on them we can trace the lines in such a way that in the end - this is a technical question - all that would exist...

JMR ... is the lines.

KM It would be a strange set of lines because they would have been infiltrated with the crumpled surface.

JMR It should be possible... who knows what it would look like. 